Research Article

\title{
Sarcocystis spp. in Romanian Slaughtered Cattle: Molecular Characterization and Epidemiological Significance of the Findings
}

\author{
Kálmán Imre $(\mathbb{D}),{ }^{1}$ Gheorghe Dărăbuş $(\mathbb{D}),{ }^{2}$ Emil Tîrziu $(\mathbb{D}),{ }^{1}$ Sorin Morariu $(\mathbb{D}),{ }^{2}$ Mirela Imre $\left(\mathbb{D},{ }^{2}\right.$ \\ Judit Plutzer $\left(\mathbb{D},{ }^{3}\right.$ Marius V. Boldea $\left(\mathbb{D},{ }^{4}\right.$ and Adriana Morar $\mathbb{D}^{1}$ \\ ${ }^{1}$ Department of Animal Production and Veterinary Public Health, Faculty of Veterinary Medicine, \\ Banat's University of Agricultural Sciences and Veterinary Medicine "King Michael I of Romania" Timişoara, \\ Timişoara 300645, Romania \\ ${ }^{2}$ Department of Parasitology and Parasitic Diseases, Faculty of Veterinary Medicine, \\ Banat's University of Agricultural Sciences and Veterinary Medicine "King Michael I of Romania" Timişoara, \\ Timişoara 300645, Romania \\ ${ }^{3}$ National Public Health Center, Department of Water Hygiene, Budapest 1097, Hungary \\ ${ }^{4}$ Department of Soil Science Mathematics and Statistics, Faculty of Agriculture, \\ Banat's University of Agricultural Sciences and Veterinary Medicine "King Michael I of Romania" Timişoara, \\ Timişoara 300645, Romania
}

Correspondence should be addressed to Kálmán Imre; kalman_imre27@yahoo.com

Received 1 August 2019; Revised 6 September 2019; Accepted 20 September 2019; Published 13 October 2019

Academic Editor: Roberto Amerigo Papini

Copyright (C) 2019 Kálmán Imre et al. This is an open access article distributed under the Creative Commons Attribution License, which permits unrestricted use, distribution, and reproduction in any medium, provided the original work is properly cited.

Species of the genus Sarcocystis are recognized as protozoan parasites infecting a wide range of animals, including humans. This study aimed to provide data on the occurrence, genetic characterization, and epidemiological significance of Sarcocystis spp. in cattle destined for human consumption in Romania. A total of 117 heart samples from slaughtered cattle in three southwestern Romanian counties (Dolj, Timiş, and Gorj) were analyzed in order to detect sarcocysts, using fresh examination microscopic techniques. Subsequently, the isolated sarcocysts and/or cyst fragments (5-15 per sample) from each infected animal were molecularly characterized. Overall, 17.9\% (21/117) of the tested animals were found to be Sarcocystis spp. positive by microscopy. Genetic characterization of Sarcocystis spp. isolates, based on sequence analysis of the $18 S$ rRNA gene, showed the presence of a single species, namely S. cruzi. No correlation was found $(p>0.05)$ between $S$. cruzi infection and the origin, age, breed, and gender of cattle, but the grazing farming system was positively associated $(p=0.031)$ with the pathogen prevalence and can be considered a risk factor $(\mathrm{OR}=3.6)$ in acquiring infection. To evaluate the possible public health risk, further investigation focused on the processing of other Sarcocystis-specific tissue matrices and evidence of human infections is recommended. This is the first study of bovine Sarcocystis infection in Romania.

\section{Introduction}

Sarcocystis spp. are common protozoan (Apicomplexa: Sarcocystidae) parasites, which, during their life cycle, require both definitive (i.e., carnivores and primates including humans) and intermediate (i.e., herbivores and omnivores) hosts $[1,2]$.

Results of molecular investigations, based on analysis of $18 S$ rRNA, cox1, and ITS1 gene sequences, have shown that cattle, as intermediate hosts, can usually harbor tissue cysts (sarcocysts) of five Sarcocystis species, including S. cruzi, S. hirsuta, S. hominis, S. rommeli (formerly known as $S$. sinensis-like), and S. heydorni [3]. Among them, S. hominis and $S$. heydorni are human pathogens. Canids act as definitive hosts for $S$. cruzi and felids for S. hirsuta and $S$. rommeli [4], while humans or other primates for S. hominis and S. heydorni [2-4]. Of the three genetic markers, only the $18 S$ rRNA gene sequences are available in GenBank for all the five Sarcocystis species known to be responsible for bovine sarcocystosis. 
Naturally, infections occurring in cattle have been occasionally associated with eosinophilic myositis [5, 6], fatal eosinophilic myocarditis [7], and economic losses (e.g., reduced milk production, abortion, or neonatal mortality). The infected intermediate hosts can harbor thin-walled ( $S$. cruzi and S. heydorni), and/or thick-walled (S. hominis, S. hirsuta, and S. rommeli) muscle sarcocysts, easily differentiable with ultrastructural microscopy [3]. The disease in humans, as a consequence of the ingestion of raw and/or undercooked meat, can occur under two forms. The first is the well known and studied intestinal sarcocystosis caused by zoonotic species (e.g., S. hominis, S. heydorni, or $S$. suihominis), resulting in symptomatic gastroenteritis, vomiting, nausea, and abdominal pain [3]. The other is the less studied muscular sarcocystosis, occurring when humans become dead-end hosts after the accidental ingestion of sporocysts from nonhuman Sarcocystis spp. [8, 9].

Epidemiological surveys of Sarcocystis spp. in cattle tissues destined to human consumption, using molecular tools, are still lacking in the majority of European countries, including Romania. Results of the few available studies conducted in Italy [10], Germany [11], and Hungary [2], aiming to assess the risk of zoonotic infections, have revealed the dominance of $S$. cruzi, but in each of them, the wellknown human pathogen S. hominis has also been reported. According to European Union legislation, the screening for sarcocysts within the meat inspection is not mandatory. This aspect can greatly contribute to the poor understanding of the parasite spreading within the bovine populations at continental level. These information gaps have been underlined by the European Food Safety Authority (EFSA) within a scientific report, encouraging the monitoring of Sarcocystis parasite presence in animals and foodstuffs by each state member [12].

Taking these into consideration, the present study aimed to provide data on the occurrence, molecular characterization, and epidemiological significance of Sarcocystis spp. from slaughtered cattle in Romania.

\section{Materials and Methods}

The study was conducted in three southwestern (between $46^{\circ} 06^{\prime} \mathrm{N}, 20^{\circ} 15^{\prime} \mathrm{E}$ and $43^{\circ} 45^{\prime} \mathrm{N}, 26^{\circ} 00^{\prime} \mathrm{E}$ ) Romanian counties (Dolj, Timiş, and Gorj), with a temperate-continental climate, from March 2017 to July 2018, covering an area of about $21.713 \mathrm{~km}^{2}$, with a total of 65.514 bovine population. A total of 117 whole heart specimens, reported as being the most relevant predilection sites for the assessment of isolation frequency of Sarcocystis spp. [13-15], were collected from slaughtered cattle during veterinary postmortem inspections in two regional slaughterhouses of the screened area. Individual animal-related (age, breed, and gender) and epidemiological (origin and farming system) data (see Table 1) were provided by veterinarians. Cattle from grazing and intensive management systems were examined. The grazing system in the screened region includes cattle form backyard and/or small-scale integrated livestock farms (up to 20-30 heads), from rural areas, in which the animals grazing on rangeland during warm season, with daily access of the possible sporocysts contaminated pastures, fodder, and drinking water. Cattle from intensively managed farming systems (more than 100 heads), with implemented biosecurity practices, generally have a lower access to the excretion of definitive hosts compared with grazing cattle. Before subjecting them to laboratory processing, the samples were stored frozen at $-20^{\circ} \mathrm{C}$.

On examination day, the hearts were thawed at room temperature. In order to detect sarcocysts, ten grams of myocardium (vertex) was processed, using the fresh examination microscopic technique [1].

In brief, the tested samples were minced in a blender, adding $40 \mathrm{ml}$ of peptone buffered solution (PBS), and stirred for $10 \mathrm{~min}$ on a magnetic stirrer for a good homogenization. Next, the mixture was poured through a sieve, collected in a $50 \mathrm{ml}$ tube and centrifuged for $5 \mathrm{~min}$, at $600 \times \mathrm{g}$. Subsequently, the supernatant was withdrawn and the resulted final concentrated pellet was resuspended in $25 \mathrm{ml}$ PBS. Finally, the pellet was transferred into a Petri dish and examined using light microscopy (100x magnification) in order to identify the possible sarcocysts [1]. Several sarcocysts and/or cyst fragments (5-15) from each microscopically positive sample were collected in microcentrifuge tubes $(500 \mu \mathrm{l})$, containing sterile distilled water $(100 \mu \mathrm{l})$, and were stored at $-20^{\circ} \mathrm{C}$ until molecular analysis.

DNA was extracted using the Isolate II Genomic DNA kit (Bioline Reagents Limited ${ }^{\circledR}$, London, UK), according to the manufacturer's recommendations. Molecular detection of Sarcocystis spp. was accomplished through genus-specific polymerase chain reaction (PCR) targeting the highly conserved $18 S$ rRNA gene ( $\sim 915 \mathrm{bp})$. The $2 \mathrm{~L}$ forward $\left(5^{\prime}-\right.$ GGATAAACCGTGGTAATTCTATG- $3^{\prime}$ ) and the $3 \mathrm{H}$ reverse (5'-GGCAAATGCTTTCGCAGTAG- $3^{\prime}$ ) specific primer set and cycling parameters were used as previously reported [16]. The positive control, which consisted of a good-quality genomic DNA extracted from a single and previously GenBankdeposited (KX008292.1) sarcocysts, together with a negative control, represented by sterile deionised water instead of DNA in the PCR mix, was also included in the reactions. Aliquots of amplified PCR products were analyzed on a $2.2 \%$ agarose gel stained with Midori Green ${ }^{\mathrm{TM}}$ (Nippon Genetics ${ }^{\circledR}$; Europe Gmbh). Subsequently, species identification was performed through bidirectional sequencing of the purified (Isolate II PCR and Gel Kit, Bioline ${ }^{\circledR}$ ) amplicons by the Macrogen Europe ${ }^{\circledR}$ Company (Amsterdam, the Netherlands). Finally, Basic Local Alignment Search Tool (BLAST) analysis was performed, in order to compare the obtained sequences to those available in the GenBank ${ }^{\circledR}$ dataset. Two S. cruzi sequences were submitted to GenBank ${ }^{\circledR}$ (accession numbers MH223459 and $\mathrm{MH} 223460)$.

Subsequently, a phylogenetic tree was constructed using the Neighbor-Joining algorithm implemented in ClustalW, based on evolutionary distances calculated by the Kimura two-parameter model with 1,000 bootstrap sampling (available online: http://clustalw.ddbj.nig.ac.jp/index.php? lang=en).

The statistical analysis of the variation of Sarcocystis spp. prevalence in cattle, in relation to the recorded animal and 
TABLE 1: Distribution of Sarcocystis cruzi in the southwestern Romanian slaughtered cattle according to individual animal and epidemiological data.

\begin{tabular}{|c|c|c|c|c|c|}
\hline $\begin{array}{l}\text { Individual animal and } \\
\text { epidemiological data }\end{array}$ & $\begin{array}{c}\text { No. of sampled } \\
\text { animals }\end{array}$ & $\begin{array}{c}\text { No. of positive } \\
\text { samples (\%) }\end{array}$ & 95\% C.I. (lower-upper) & $\begin{array}{c}\text { Odds ratios } \\
(\mathrm{OR})(95 \% \mathrm{CI}) \\
\end{array}$ & $p$ value \\
\hline \multicolumn{6}{|l|}{ Counties } \\
\hline Dolj & 47 & $7(14.9)$ & $6.7-28.9$ & Reference & 0.643 \\
\hline Timiş & 30 & $5(16.7)$ & $6.3-35.5$ & $0.9(0.3-3.1)$ & \\
\hline Gorj & 40 & $9(22.5)$ & $11.4-38.9$ & $1.5(0.4-4.9)$ & \\
\hline \multicolumn{6}{|l|}{ Age group (years) } \\
\hline$\leq 2$ & 31 & $3(9.7)$ & $2.5-26.9$ & Reference & 0.381 \\
\hline 2 to 8 & 50 & $10(20.0)$ & $10.5-34.1$ & $2.3(0.6-9.3)$ & \\
\hline$>8$ & 36 & $8(22.2)$ & $10.7-39.6$ & $2.7(0.6-11.1)$ & \\
\hline \multicolumn{6}{|l|}{ Breed } \\
\hline Holstein Friesian & 32 & $5(15.6)$ & $5.9-33.6$ & Reference & 0.875 \\
\hline Bălţată Românească & 24 & $4(16.7)$ & $5.5-38.3$ & $1.1(0.3-4.5)$ & \\
\hline Crossbreed & 61 & $12(19.7)$ & $11.0-32.2$ & $1.3(0.4-4.2)$ & \\
\hline \multicolumn{6}{|l|}{ Gender } \\
\hline Female & 102 & $18(17.7)$ & $11.1-26.7$ & Reference & 0.825 \\
\hline Male & 15 & $3(20.0)$ & $5.3-48.6$ & $0.9(0.2-3.4)$ & \\
\hline \multicolumn{6}{|l|}{ Farming system } \\
\hline Intensive & 48 & $4(8.3)$ & $2.7-20.9$ & Reference & 0.031 \\
\hline Grazing & 69 & $17(24.6)$ & $15.4-36.7$ & $3.6(1.1-11.5)$ & \\
\hline Total & 117 & $21(17.9)$ & $11.7-26.3$ & & \\
\hline
\end{tabular}

epidemiological data, was performed with Pearson's chisquare $(\chi 2)$ test, using IBM SPSS Statistics for Windows, Version 21.0 (IBM Corp., Armonk, NY, USA). A $p$ value $\leq 0.05$ was considered significant. In addition, risk factors were evaluated, using the multivariable regression analysis. Each variable was included in the binary logit model as an independent variable, resulting in the calculation of odds ratios (ORs), with 95\% confidence intervals (CIs).

\section{Results}

The results of the survey are summarized in Table 1. Overall, a total of 21 out of 117 (17.9\%) slaughtered southwestern Romanian cattle were found to be Sarcocystis positive, by microscopically fresh examination. The observed elongated ovals (up to $300 \mu \mathrm{m}$ long and $110 \mu \mathrm{m}$ wide; [15]) and spherical sarcocysts had a smooth and thin wall, enclosing numerous bradyzoites (Figure 1). These observations were suggestive for $S$. cruzi and/or $S$. heydorni species [3, 15]. Sarcocystis-infected animals were recorded in all the screened counties (Dolj: 14.9\%; Timiş: 16.7\%; Gorj: 22.5\%), of all ages ( $\leq 2$ years: $9.7 \% ; 2$ to 8 years: $20 \%$; $>8$ years: $22.2 \%$ ) and breeds (Holstein Friesian: 15.6\%; Bălţată Românească: 16.7\%; crossbreed: 19.7\%) (Table 1), but the registered prevalence of the infection did not reach the level of statistical significance $(p>0.05)$. The numbers of infected female and male cattle did not differ significantly from each other $(17.7 \%$ vs. $20.0 \%)$. However, the cattle reared in the grazing system $(24.6 \%, \mathrm{OR}=3.6,95 \%$ CI $1.1-11.5)$ were found to be more susceptible to Sarcocystis infection than those farmed in the intensive system $(8.3 \%, 95 \%$ CI 2.7-20.9, $p=0.031$ ).

Molecular characterization of Sarcocystis isolates based on sequence analysis of the $18 S$ rRNA gene was successfully

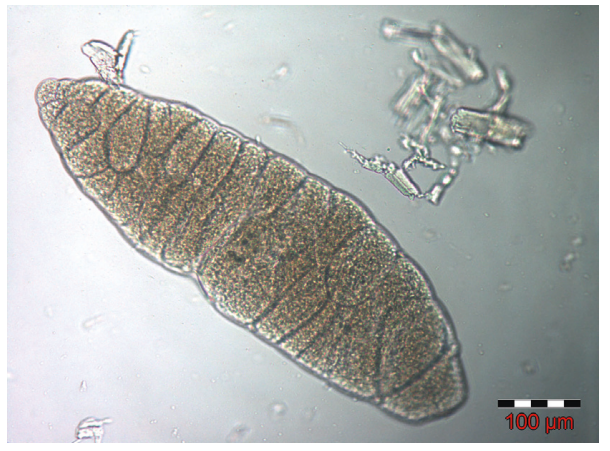

FIGURE 1: Light microscope appearance (100x magnification) of the thin-walled $S$. cruzi with several internal septa that form compartments containing bradyzoites.

done in all microscopically positive specimens and indicated that all 21 isolates were positive for S. cruzi (with 97-100\% homology to GenBank ${ }^{\circledR}$ reference sequences; KT901168.1, JX679467.1, AB682781.1). Analysis of the phylogenetic relationships of our sequences and other GenBank ${ }^{\circledR}$-retrieved Sarcocystis reference sequences recorded in cattle in different countries and/or reported in scientific papers (see Figure 2) showed that our isolates clustered closely with other S. cruzi sequences isolated from cattle in Argentina, China, Egypt, Japan, and Uruguay.

\section{Discussion}

To the authors' knowledge, this is the first molecular study of bovine Sarcocystis infection in Romania, providing information for the nation and mainland Europe. Contrary to the high overall Sarcocystis infection prevalence in cattle registered in other studies $(66.2 \%,[2] ; 78.1 \%$, [10]; 100\%, 


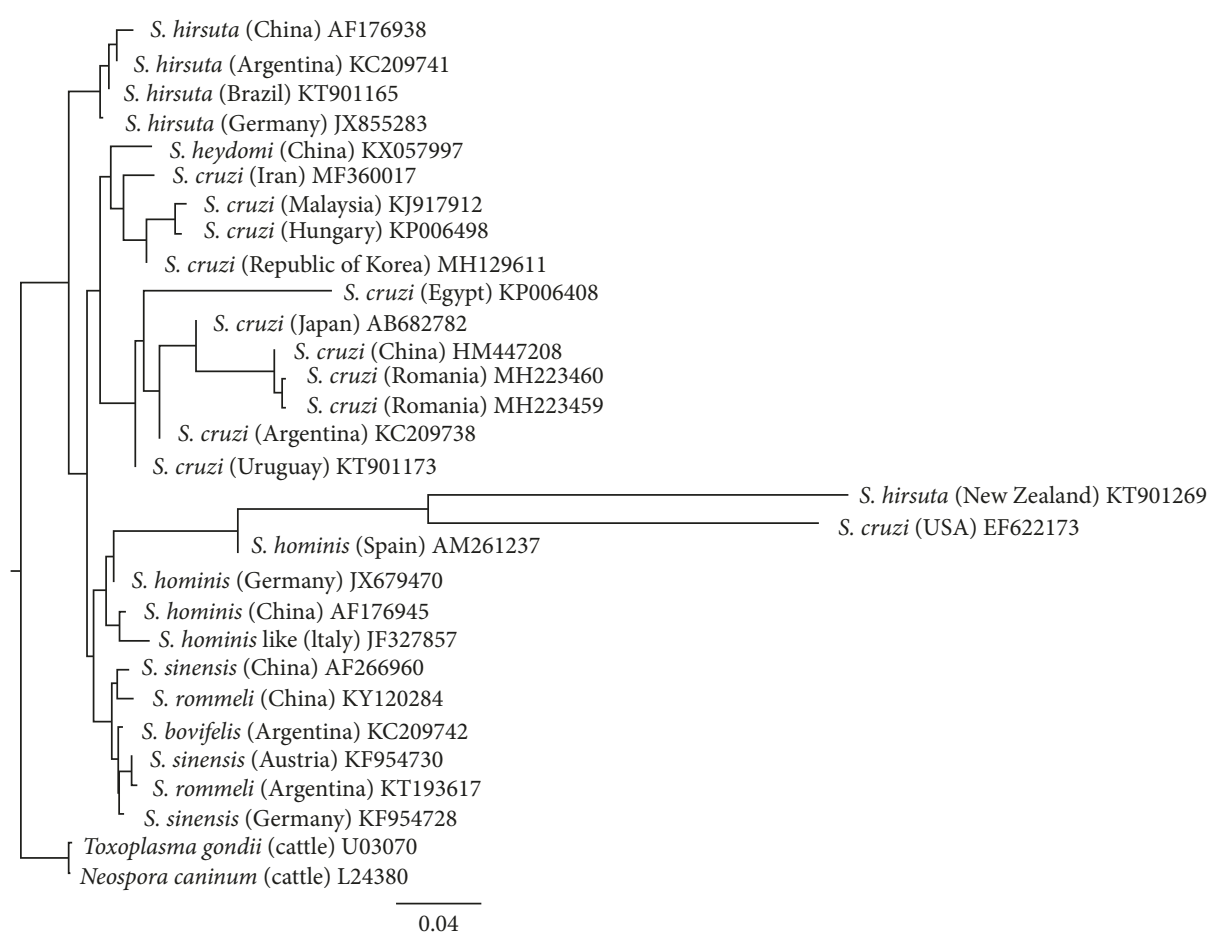

Figure 2: Phylogenetic tree showing the relationship of the S. cruzi sequences resulting from the current survey (marked with bold), and other cattle-specific Sarcocystis spp. reported in different countries (in brackets), based on analysis of a partial sequence of the $18 S$ rRNA gene.

$[17,18] ; 41.5 \%,[19] ; 97.4 \%,[20])$, in our survey, a considerably lower infection level (17.9\%) was recorded. However, it should be taken into account that in some another investigations, a larger amount of tissue samples were processed (e.g., $[17,19,20]$ ), and the registered cumulative prevalence values are the result of the screening of at least two Sarcocystis-specific tissue matrices (e.g., heart, skeletal muscle, esophagus, diaphragm, and tongue $[2,10,18]$ ), with different diagnosis methods (e.g., light microscopy and fresh, pepsin digestion, and transmission electron microscope examinations), under different combination forms. Likewise, in our study, the direct PCR screening of the final concentrated pellet or heart tissue of the microscopically negative samples, which may increase the detection sensitivity of Sarcocystis spp., as highlighted by Pritt et al. [21], would have contributed to the diagnostic precision. Moreover, the pellet screening with a higher power objective lens (e.g., 400x), like in the case in [17], would facilitate the identification of bradyzoites resulted from the ruptured cysts, thus increasing the prevalence of infection. Taking these into consideration, it can be assumed that in our study, the true prevalence is underestimated. Similar to our findings, the exclusive presence or dominance of the nonzoonotic $S$. cruzi in cattle tissues has been genetically confirmed in other studies conducted in Brazil (78 successfully molecularly characterized samples out of 78 , [17]), Republic of Korea (31/31, [15]), Malaysia (17/17, [18]), Republic of China (215/216, [19]), Argentina (24/29, [1]), Hungary (23/36, [2]), Italy (285/384, [10]), Germany (134/ 257, [11]), and Iran (89/90, [22]), highlighting that this species is the most prevalent in cattle worldwide. However, caution should be taken in interpreting molecular survey results because the differences in the registered Sarcocystis spp. can be markedly influenced by the processed tissue matrices (e.g., myocardium, striated muscle, esophagus, diaphragm, and tongue). In this regard, the presence of the only species in the current survey is not surprising, considering that until now, the evidence of two other common Sarcocystis spp., namely, S. hirsuta and S. hominis, has not been molecularly confirmed in cattle myocardium samples [23]. Likewise, 17 out of 21 (81\%) S. cruzi sequences have been isolated from cattle reared under the grazing system. Considering that domestic dogs (Canis lupus familiaris) are recognized as natural definitive hosts for $S$. cruzi, the intense usage of these carnivores in cattle management in grazing systems in our country results in a close relationship between the definitive and intermediate hosts. In addition, the free access of wild carnivores to pastures can favor the cattle contamination with the infective stages of the parasite. This could provide another explanation for the exclusive detection of this species in the current survey [18]. The complete life cycle of $S$. hominis and S. hirsuta in cattle requires the ingestion of sporocysts shed by primates and cats [23], a consequence of improper management of human sewage and environmental occurrence of unburied cat feces [18].

From the epidemiological point of view, based on the not-so-negligible prevalence $(17.9 \%)$ found in cattle, the results depose for an environmental contamination with $S$. cruzi sporocysts, shed by infected carnivores. The sporocysts 
can resist and retain their infectivity in the environment for different external factors (e.g., freezing, high temperature, and several disinfectants), for a long period [23]. Therefore, the ubiquity of cattle exposure suggests that human beings also may ingest sporocysts of S. cruzi. Indeed, a S. cruzipositive diarrheal sample was identified by microscopic and molecular examination in an AIDS-affected woman in Iran [24]. However, the clinical consequence of such exposure remains entirely unexplored. Likewise, the study provides useful insights for veterinarians regarding the importance of $S$. cruzi in bovine health populations in Romania. For instance, $S$. cruzi appears to be able to cause abortions that are clinically indistinguishable from those caused by Neospora and differentiation depends on immunohistochemistry or PCR [25]. In addition, $S$. cruzi is strongly suspected to be implicated in cases of fulminant death $[2,26]$, as well as in cases of eosinophilic myositis in cattle [7].

No correlation was found between the Sarcocystis infection positivity and the origin, age, breed, and gender of cattle, but the grazing farming system was positively associated ( $p=0.031$ ) with the pathogen prevalence and can be considered a risk factor $(\mathrm{OR}=3.6)$ in acquiring the infection. Thereby, a preliminary comparison of cattle husbandry indicates differential exposure to $S$. cruzi, a parasite excreted by dogs, resulting in a prevalence in grazed cattle, at least $10 \%$ greater. However, caution should be taken in interpreting the statistical results of the study because the limited number of processed samples can increase the margin of error and decrease the power of the analysis in discerning meaningful differences between the enrolled variables. According to our results, in other epidemiological surveys aimed to detect Sarcocystis infection in cattle, gender- and breed-related susceptibility to infection was not demonstrated (reviewed by [23]). Contrary to our findings, in a study conducted in Tunisia, Amairia et al. [27] reported a significantly higher $(p=0.05) S$. cruzi detection rate in animals aged between 2 and 8 years, compared to young animals. The same tendency has been observed by Yang et al. [19] in cattle older than 2 years in a survey conducted in central China. Moré et al. [14] have also reported higher values of sarcocysts per gram in the myocardium of Argentinean adult cattle, compared to young cattle. The longer and repetitive exposure of adult animals to infective sporocysts, compared to young ones, can support these findings.

The grazing farming system has a positive effect on the risk of $S$. cruzi infection. This observation can be sustained by the more intense perpetuation of the intermediate and definitive host relationship within the parasite's life cycle in this system, compared to the industrial one, based on the free access of cattle to infective forms of the parasites shed by definitive hosts (e.g., domestic and/or wild carnivores) [17]. Dogs excreting infective sporocysts contaminate the natural environment of grazing cattle, including feeders with silage and hay, the pasture, and water sources. Evidence of the parasite in cattle from the industrial system with restricted access for dogs may be related to the vehiculation of infective forms of the parasite in flies, which can transport the parasite from dog feces to cattle feed, as has been highlighted by
Markus [28]. In addition, feeding alleys, stored grass, corn silage, and water contamination with sporocysts, due to management failures (e.g., accidental access of dogs kept in the neighborhood of farms), can also be considered a source of infection for cattle in the intensive farming system.

The structure of the resulting phylogenetic tree (Figure 2) revealed that the $S$. cruzi isolates, selected for the tree construction, clustered in several branches together with other cattle-specific and GenBank-deposited Sarcocystis species ( $S$. heydorni and $S$. hirsuta) sequences from various parts of the world, a phenomenon which has been noted previously [29]. In the last decade, even if other more distinct sequences have subsequently been identified worldwide, the reported new data remain supportive of global dissemination of at least one invariant strain, perhaps with more diversity in Malaysia, in accordance with the findings published by $\mathrm{Ng}$ et al. [18]. It is also possible that more distinct sequences may eventually be attributed to other parasite species.

\section{Conclusions}

The results of this study highlighted that Romanian cattle destined for human consumption can harbor S. cruzi sarcocysts, with uniform distribution in the screened area, and the grazing farming system can be considered a risk factor in bovine sarcocystosis. The study provides useful information for veterinarians about the epidemiology of Sarcocystis infecting cattle in our country, although, for a possible public health hazard evaluation, a larger number of animals and other Sarcocystis-specific tissue matrices need to be screened. Likewise, investigations that highlight the presence of infections in humans are necessary for a better understanding of the zoonotic risk of sarcocystosis.

\section{Data Availability}

The sequences (accession numbers MH223459 and MH223460) used to support the findings of this study have been deposited in the GenBank repository. The datasets generated and analyzed during the current study are included within the article.

\section{Conflicts of Interest}

The authors declare that there are no conflicts of interest regarding the publication of this paper.

\section{Authors' Contributions}

Kálmán Imre and Mirela Imre have contributed equally.

\section{Acknowledgments}

The authors highly appreciate the indispensable contribution of Ing. Anca Deac and veterinary student Florin Tufiş during sample collection and processing. The work was funded by the Internal Competition for Research (grant no. 2756/2015) of the Banat University of Agricultural Sciences and Veterinary Medicine "King Michael I of Romania" from 
Timișoara, Romania, obtained by Kálmán Imre, and was also partially supported by the POSCCE Project SMIS no. 2669.

\section{References}

[1] G. Moré, P. Abrahamovich, S. Jurado et al., "Prevalence of Sarcocystis spp. in Argentinian cattle," Veterinary Parasitology, vol. 177, no. 1-2, pp. 162-165, 2011.

[2] S. Hornok, A. Mester, N. Takács et al., "Sarcocystis-infection of cattle in Hungary," Parasites and Vectors, vol. 8, no. 1, p. 69, 2015.

[3] J. P. Dubey, "Foodborne and waterborne zoonotic sarcocystosis," Food and Waterborne Parasitology, vol. 1, no. 1, pp. 2-11, 2015.

[4] J. P. Dubey, G. Moré, E. van Wilpe, R. Calero-Bernal, S. K. Verma, and G. Schares, "Sarcocystis rommeli, n. sp. (Apicomplexa: Sarcocystidae) from cattle (Bos taurus) and its differentiation fromSarcocystis hominis," Journal of Eukaryotic Microbiology, vol. 63, no. 1, pp. 62-68, 2016.

[5] W. Wouda, J. J. Snoep, and J. P. Dubey, "Eosinophilic myositis due to Sarcocystis hominis in a beef cow," Journal of Comparative Pathology, vol. 135, no. 4, pp. 249-253, 2006.

[6] L. Vangeel, K. Houf, P. Geldhof et al., "Different Sarcocystis spp. are present in bovine eosinophilic myositis," Veterinary Parasitology, vol. 197, no. 3-4, pp. 543-548, 2013.

[7] V. Aráoz, C. da Silva Silveira, G. Moré, G. Banchero, F. RietCorrea, and F. Giannitti, "Fatal Sarcocystis cruzi-induced eosinophilic myocarditis in a heifer in Uruguay," Journal of Veterinary Diagnostic Investigation, vol. 31, no. 4, pp. 656660, 2019.

[8] R. Murata, J. Suzuki, A. Hyuga, T. Shinkay, and K. Sadamasu, "Molecular identification and characterization of Sarcocystis spp. in horsemeat and beef marketed in Japan," Parasite, vol. 25 , no. $27,2018$.

[9] B. M. Rosenthal, "Sarcocystosis," in Hunter's Tropical Medicine and Emerging Infectious Disease Chapter 103, pp. 780-783, Elsevier, Amsterdam, Netherlands, 9th edition, 2013.

[10] L. Domenis, S. Peletto, L. Sacchi et al., "Detection of a morphogenetically novel Sarcocystis hominis-like in the context of a prevalence study in semi-intensively bred cattle in Italy," Parasitology Research, vol. 109, no. 6, pp. 1677-1687, 2011.

[11] G. Moré, A. Pantchev, J. Skuballa et al., "Sarcocystis sinensis is the most prevalent thick-walled Sarcocystis species in beef on sale for consumers in Germany," Parasitology Research, vol. 113, no. 6, pp. 2223-2230, 2014.

[12] M. A. Taylor, J. Boes, P. Boireau et al., "Developent of harmonised schemes for the monitoring and reporting of Sarcocystis in animals and foodstuffs in the European Union," 2010, http://www.efsa.europa.eu/en/supporting/pub/33e.htm.

[13] M. Fukuyo, G. Battsetseg, and B. Byambaa, "Prevalence of Sarcocystis infection in meat-producing animals in Mongolia," Southeast Asian Journal of Tropical Medicine and Public Health, vol. 33, no. 3, pp. 490-495, 2002.

[14] G. Moré, W. Basso, D. Bacigalupe, M. C. Venturini, and L. Venturini, "Diagnosis of Sarcocystis cruzi, Neospora caninum, and Toxoplasma gondii infections in cattle," Parasitology Research, vol. 102, no. 4, pp. 671-675, 2008.

[15] T.-I. Choi, E.-J. Hong, S.-Y. Ryu et al., "Detection and identification of Sarcocystis cruzi (Protozoa: apicomplexa) by molecular and ultrastructural studies in naturally infected Korean cattle (Bos taurus coreanae) from daejeon, Korea,"
The Korean Journal of Parasitology, vol. 56, no. 2, pp. 121-127, 2018.

[16] Z. Yang, Y. X. Zuo, Y. G. Yao, X. W. Chen, G. C. Yang, and Y. P. Zhang, "Analysis of the $18 \mathrm{~S}$ rRNA genes of Sarcocystis species suggests that the morphologically similar organisms from cattle and water buffalo should be considered the same species," Molecular and Biochemical Parasitology, vol. 115, no. 2, pp. 283-288, 2001.

[17] M. S. T. Ferreira, F. S. F. Vogel, L. A. Sangioni et al., "Sarcocystis species identification in cattle hearts destined to human consumption in southern Brazil," Veterinary Parasitology: Regional Studies and Reports, vol. 14, pp. 94-98, 2018.

[18] Y. H. Ng, M. Y. Fong, V. Subramaniam, S. Shahari, and Y. L. Lau, "Short communication: genetic variants of Sarcocystis cruzi in infected Malaysian cattle based on $18 \mathrm{~S}$ rDNA," Research in Veterinary Science, vol. 103, pp. 201-204, 2015.

[19] Y. Yang, H. Dong, R. Su et al., "High prevalence of Sarcocystis spp. infections in cattle (Bos taurus) from central China," Parasitology International, vol. 67, no. 6, pp. 800-804, 2018.

[20] L. Vangeel, K. Houf, K. Chiers, J. Vercruysse, K. D'herde, and R. Ducatelle, "Molecular-based identification of Sarcocystis hominis in Belgian minced beef," Journal of Food Protection, vol. 70, no. 6, pp. 1523-1526, 2007.

[21] B. Pritt, T. Trainer, L. Simmons-Arnold, M. Evans, D. Dunams, and B. M. Rosenthal, "Detection of Sarcocystis parasites in retail beef: a regional survey combining histological and genetic detection methods," Journal of Food Protection, vol. 71, no. 10, pp. 2144-2147, 2008.

[22] M. Akhlaghi, M. Razavi, and A. Hosseini, "Molecular differentiation of bovine sarcocysts," Parasitology Research, vol. 115, no. 7, pp. 2721-2728, 2016.

[23] J. P. Dubey, R. Calero-Bernal, B. M. Rosenthal, C. A. Speer, and R. Fayer, Sarcocystosis of Animals and Humans, CRC Press, Boca Raton, FL, USA, 2nd edition, 2016.

[24] M. Agholi, S. N. Shahabadi, M. H. Motazedian, and G. R. Hatam, "Prevalence of enteric protozoan oocysts with special reference to Sarcocystis cruzi among fecal samples of diarrheic immunodeficient patients in Iran," The Korean Journal of Parasitology, vol. 54, no. 3, pp. 339-344, 2016.

[25] T. J. Parkinson, "Specific infectious diseases causing infertility and subfertility in cattle," in Veterinary Reproduction and Obstetrics: Section IV: Subfertility, D. E. Noakers, T. J. Parkinson, and G. C. W. England, Eds., pp. 434-466, Elsevier, Amsterdam, Netherlands, 10th edition, 2019.

[26] P. F. Freiler, I. G. Mayhew, and R. Pollock, "Bovine sarcocystiosis: pathologic features of naturally occurring infection with Sarcocystis cruzi," American Journal of Veterinary Research, vol. 40, no. 5, pp. 651-657, 1979.

[27] S. Amairia, Y. Amdouni, M. R. Rjeibi, M. Rouatbi, S. Awadi, and M. Gharbi, "First molecular detection and characterization of Sarcocystis species in slaughtered cattle in NorthWest Tunisia," Meat Science, vol. 122, pp. 55-59, 2016.

[28] M. B. Markus, "Flies as natural transport hosts of Sarcocystis and other coccidia," Journal of Parasitology, vol. 66, no. 2, pp. 361-362, 1980.

[29] B. M. Rosenthal, D. B. Dunams, and B. Pritt, "Restricted genetic diversity in the ubiquitous cattle parasite, Sarcocystis cruzi," Infection, Genetics and Evolution, vol. 8, no. 5, pp. 588-592, 2008. 


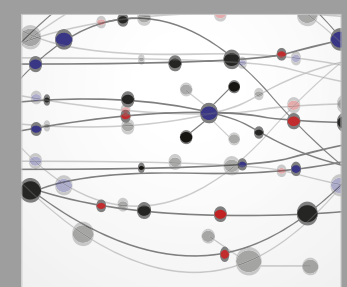

The Scientific World Journal
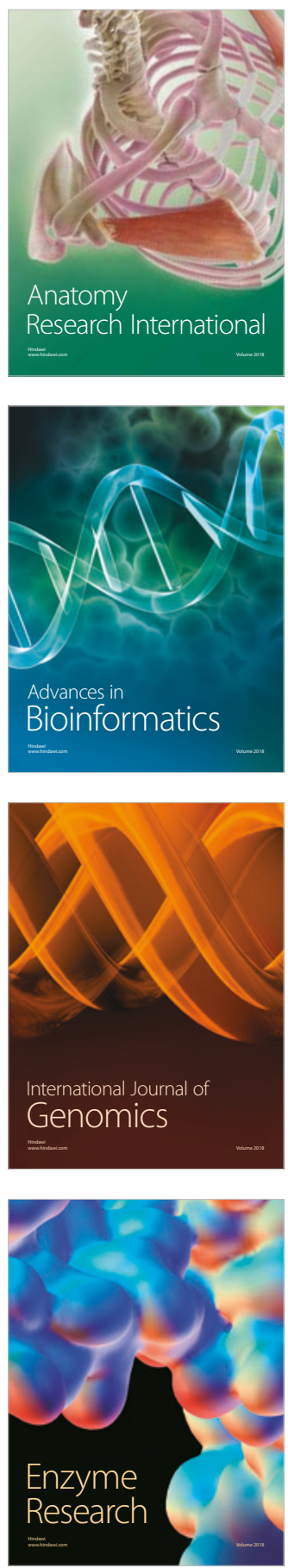
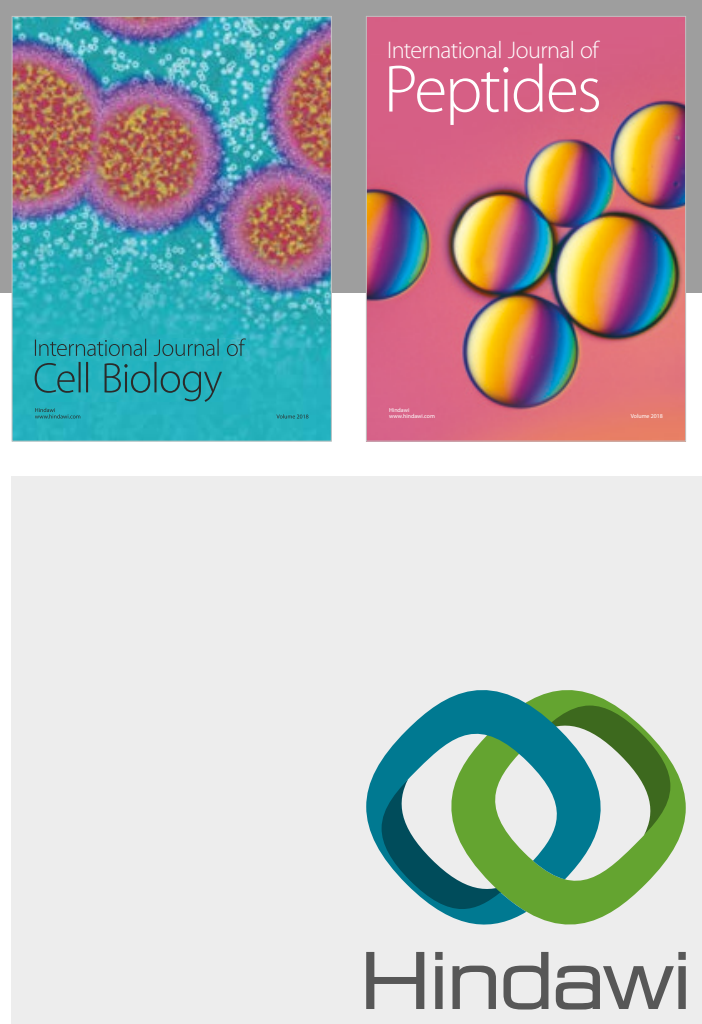

Submit your manuscripts at

www.hindawi.com
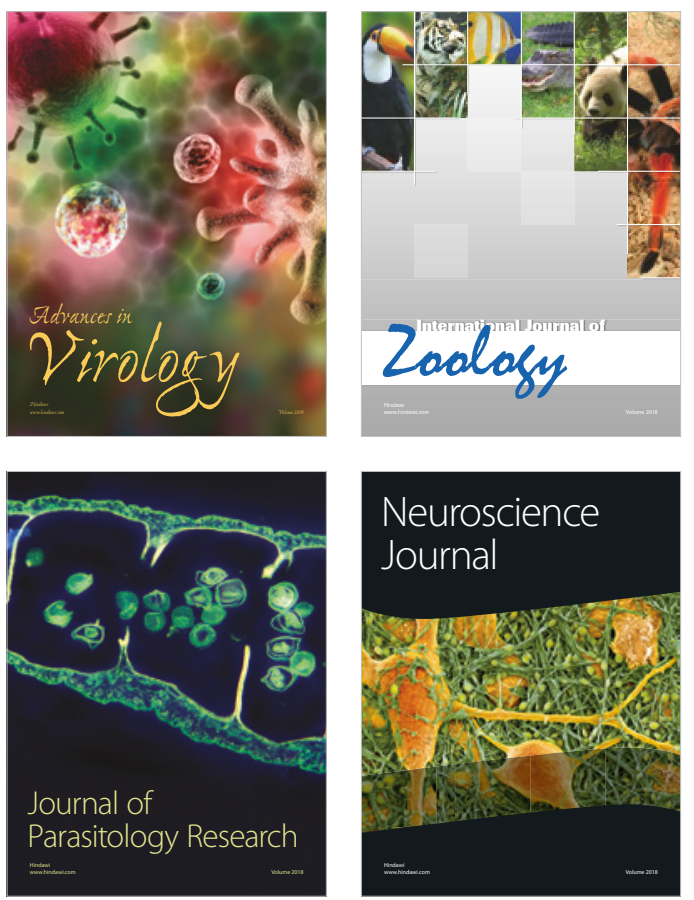
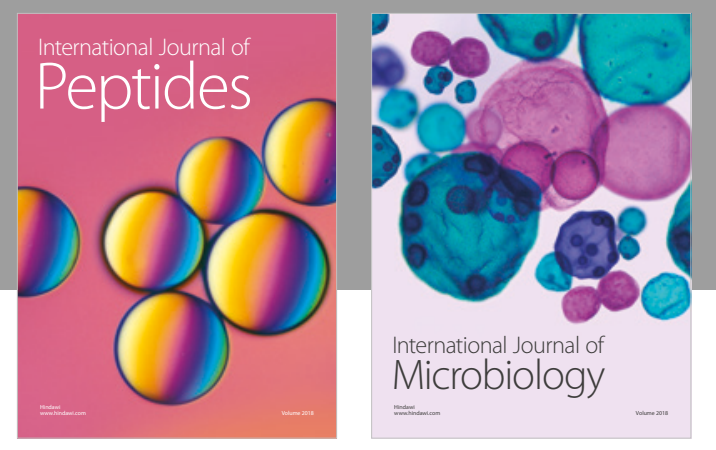

nternational Journal of Microbiology
Journal of
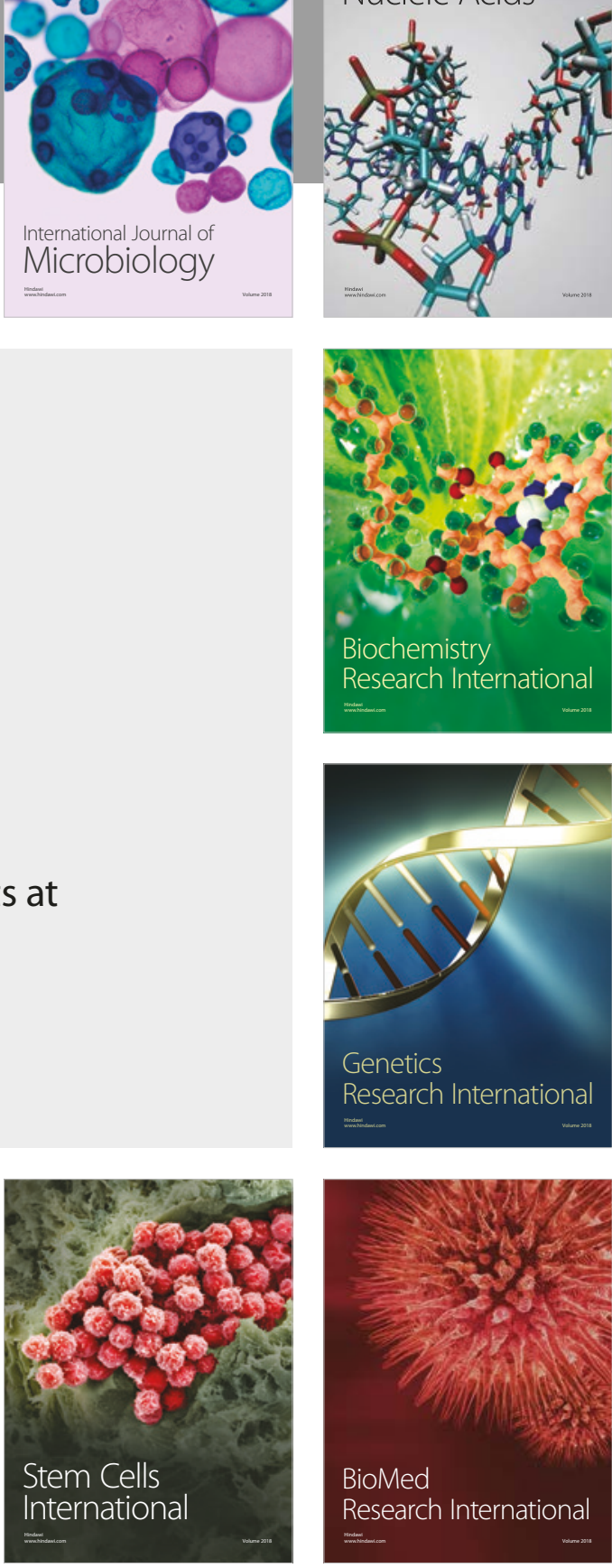
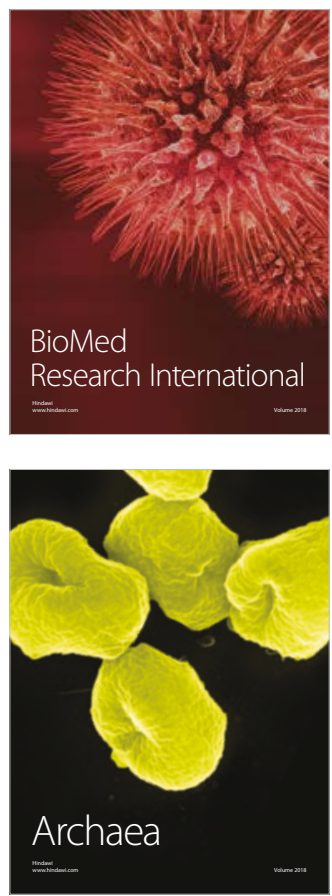